\title{
Radiation-Induced Fibrous Mediastinitis
}

National Cancer Institute

\section{Source}

National Cancer Institute. Radiation-Induced Fibrous Mediastinitis. NCI Thesaurus. Code C27180.

Fibrous mediastinitis arising due to radiation therapy. 